\title{
Characterization of EEG patterns in brain-injured subjects and controls after a Snoezelen ${ }^{\circledR}$ intervention
}

Carlos Gómez ${ }^{\mathrm{a}, *}$, Jesús Poza ${ }^{\mathrm{a}, \mathrm{b}}$, María T. Gutiérrez ${ }^{\mathrm{c}}$, Esther Prada ${ }^{\mathrm{c}}$, Nuria Mendoza $^{\mathrm{d}}$, and Roberto Hornero ${ }^{\mathrm{a}, \mathrm{b}}$

a Biomedical Engineering Group, Universidad de Valladolid, Valladolid, Spain.

b IMUVA, Instituto de Investigación en Matemáticas, Universidad de Valladolid, Valladolid, Spain.

c Centro de Referencia Estatal (CRE) para la Atención a Personas con Grave Discapacidad y Dependencia, San Andrés del Rabanedo (León), Spain.

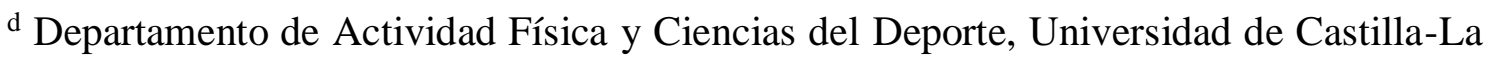
Mancha, Toledo, Spain.

* Corresponding author: Carlos Gómez

Biomedical Engineering Group, E.T.S. Ingenieros de Telecomunicación, Universidad de Valladolid, Campus Miguel Delibes, Paseo Belén 15, 47011 - Valladolid, Spain.

Tel. +34983423981

E-mail address: carlos.gomez@tel.uva.es

URL: www.gib.tel.uva.es 
Abstract- Background and objective. The aim of this study was to assess the changes induced in electroencephalographic (EEG) activity by a Snoezelen ${ }^{\circledR}$ intervention on individuals with brain-injury and control subjects. Methods: EEG activity was recorded preceding and following a Snoezelen ${ }^{\circledR}$ session in 18 people with cerebral palsy (CP), 18 subjects who have sustained traumatic brain-injury (TBI) and 18 controls. EEG data were analyzed by means of spectral and nonlinear measures: median frequency $(M F)$, individual alpha frequency (IAF), sample entropy (SampEn) and Lempel-Ziv complexity (LZC). Results: Our results showed decreased values for $M F, I A F$, SampEn and $L Z C$ as a consequence of the therapy. The main changes between pre-stimulation and post-stimulation conditions were found in occipital and parietal brain areas. Additionally, these changes are more widespread in controls than in brain-injured subjects, which can be due to cognitive deficits in TBI and CP groups. Conclusions: Our findings support the notion that Snoezelen ${ }^{\circledR}$ therapy affects central nervous system, inducing a slowing of oscillatory activity, as well as a decrease of EEG complexity and irregularity. These alterations seem to be related with higher levels of relaxation of the participants.

Keywords- Brain-injury, electroencephalograpy (EEG), biomedical signal processing, Snoezelen ${ }^{\circledR}$, non-pharmacological therapies. 


\section{INTRODUCTION}

Snoezelen ${ }^{\circledR}$ rooms, also termed as multi-sensory rooms, were developed in the Netherlands in 1975 [1]. The word "snoezelen" is a composite of the Dutch words "snuffelen", which means to seek out or to explore, and "doezelen", meaning to relax [2]. Snoezelen ${ }^{\circledR}$ rooms contain materials and equipment for sensory exploration, such as radios, sounds of animals or the sea, projectors, mirror balls, bubble columns, aromatherapy oils, rocking chairs, water beds, optic fiber bundles and other tactile stimulation objects $[3,4]$. The aim of combining all these materials is to promote relaxation and enjoyment in their participants. The role of the staff involved in the process is to guide and encourage the individuals to interact with the sensory equipment [3].

In the last years, there is a growing interest about Snoezelen ${ }^{\circledR}$ intervention. Particularly, several researches have focused on the use of multi-sensory environments in people with intellectual disabilities (for a review, see [5] or [6]). For instance, Singh et al. [7] suggested a reduction of aggressive acts and self-injurious behavior in individuals with mental retardation following their exposure to the Snoezelen ${ }^{\circledR}$ therapy. Other study demonstrated the effectiveness of this intervention in facilitating new skills in children with severe and multiple disability [8]. In Withers et al. [9], the benefits of a treatment based on a combination of Snoezelen ${ }^{\circledR}$ with other non-pharmacological therapies were reported: marked decrease in the subjects' self-injurious behavior, along with a general increase of positive behaviors. The growing acceptance of Snoezelen ${ }^{\circledR}$ rooms has been accompanied by several research studies to assess the effects of this treatment also in dementia patients [10-12], people with chronic pain [13], breastfeeding women [14], autistic patients [15] and children with Rett disorder [16], among others. Although negative outcomes have been revealed by some authors [15], almost all 
studies have reported a broad range of benefits, such as relaxation [14], reduction of pain [13], lower agitation [10], greater independence in activities of daily living [13], decrease of self-injurious behavior [7,8], happiness and calmness [11]. In sum, an increasing amount of evidence supports the hypothesis that multi-sensory environments generate different positive effects. However, the results of these researches are usually based on subjective observations and/or qualitative data. Therefore, further studies are needed to quantify in an objective way the effects of this non-pharmacological intervention.

In the current research, quantitative effects of Snoezelen ${ }^{\circledR}$ rooms were studied in brain-injured subjects and controls by means of EEG analyses. To our knowledge, multi-sensory intervention in this group has been applied just in a few studies. Hotz et al. [17] revealed an overall beneficial use of this therapy in children recovering from severe brain-injury, including diminished values of heart rate and muscle tone. In our previous study [18], we demonstrated that multi-sensory stimulation produces a slowing of EEG oscillatory activity in brain-injured patients and controls. This fact may indicate that Snoezelen ${ }^{\circledR}$ therapy induces a state of relaxation. The main limitation of our previous work is that a heterogeneous brain-injury group was analyzed [18]. To solve this drawback, in the current study, brain-injured subjects have been classified into people with cerebral palsy $(\mathrm{CP})$ and subjects who have sustained traumatic brain-injury (TBI). CP is a non-progressive disorder caused by brain injury before, during, or immediately after birth. It is the major physical disability affecting the functional development of children, occurring in approximately 2 in 1000 liveborn infants [19]. On the other hand, TBI is the most common cause of death and disability in young people [20]. It is estimated that 57 million people worldwide are hospitalized due to TBI, but the proportion living with TBI-related disability is unknown [21]. Causes include motor 
vehicles crashes, falls, assaults and sport activities [22]. Due to the high number of people affected by brain-injury, both CP and TBI, proper interventions are needed to increase their quality of life.

The aim of our study is to assess the quantitative effects induced in EEG activity by a Snoezelen ${ }^{\circledR}$ intervention on individuals with brain-injury and control subjects. For this purpose, EEG activity was analyzed in $18 \mathrm{CP}$ subjects, $18 \mathrm{TBI}$ individuals and 18 controls by means of spectral and nonlinear measures: median frequency $(M F)$, individual alpha frequency $(I A F)$, sample entropy (SampEn) and Lempel-Ziv complexity $(L Z C)$. These four methods provide a comprehensive description of EEG dynamics before and after the intervention. We want to test the hypothesis that Snoezelen ${ }^{\circledR}$ therapy evokes alterations in the cerebral activity of brain-injured patients and control subjects.

\section{MATERIAL}

\section{A. Subjects}

For the present study, EEG signals were obtained from 36 brain-injured subjects. This group was divided into 18 TBI subjects (15 men and 3 women with a mean age of $38.06 \pm 8.25$ years, mean \pm standard deviation, SD) and 18 participants with $\mathrm{CP}(11$ men and 7 women with a mean age of $44.61 \pm 10.89$ years). Brain-injury diagnosis was determined on the basis of exhaustive clinical examinations. Diagnostic information for both TBI and CP groups is presented in Table 1. The patients were recruited from the "Centro de Referencia Estatal para la Atención a Personas con Grave Discapacidad y Dependencia" (CRE-APGDD) at San Andrés del Rabanedo (Spain) and from “Asociación Leonesa de Daño Cerebral Sobrevenido" (León, Spain). The control group consisted of eighteen healthy volunteers ( 9 men and 9 women, mean age $=37.56 \pm$ 
5.58) without past or present neurological disorders. The populations showed no significant differences in age and gender ( $p$-values $>0.05$, Kruskal-Wallis test).

All enrolled healthy subjects, patients and patients' guardians gave written informed consent for the participation in this research study. Moreover, the study protocol was approved by the local Ethics Committee according to the code of ethics of the World Medical Association (Declaration of Helsinki).

\section{PLEASE, DISPLAY TABLE 1 AROUND HERE}

\section{B. Procedure}

This study was accomplished in the Snoezelen ${ }^{\circledR}$ room placed in CRE-APGDD. It is a white room equipped with sensory equipment to create a relaxing but also stimulating atmosphere. A therapist administered one-to-one 18 minutes treatment sessions. The therapist was involved with the subject's exposure to the environment by facilitating interaction. The Snoezelen ${ }^{\circledR}$ session was divided in four parts. In the first one, colored bubble tubes were presented to the participants. The second one was focused on optic fiber bundles. In the third block, auditory and visual sensory equipment were combined: bubble tubes, a rotating mirror ball and relaxing sounds of nature. In the last part, a projector displays a moving clouds effect, while a stereo system plays sounds of nature. Figure 1 shows a schematic representation of the session protocol, whereas a detailed description can be found in our previous study [18]. 


\section{EEG recording}

EEG signals were acquired from 19 derivations of the international 10-20 system with a Nihon Khoden Neurofax JE-921A EEG amplifier. During data acquisition, subjects were asked to remain awake, relaxed and with their eyes closed. Additionally, participants were continuously monitored during the recording period in order to prevent drowsiness. For each subject, 5 min of spontaneous EEG activity was recorded preceding and following the Snoezelen ${ }^{\circledR}$ session. EEG signals were acquired at a sampling frequency of $500 \mathrm{~Hz}$ and processed with a 0.08 to $120 \mathrm{~Hz}$ band-pass filter and a $50 \mathrm{~Hz}$ notch filter. Afterwards, an experienced technician performed offline artifact rejection. A mean of $22.56 \pm 10.06$ artifact-free epochs of $5 \mathrm{~s}$ (2500 samples) per channel and participant were selected. Finally, selected epochs were digitally band-pass filtered between 1 and $40 \mathrm{~Hz}$ [18].

\section{METHODS}

\section{A. Median frequency $(M F)$}

To characterize the spectral content of EEG data, the Fourier transform was used. Initially, we computed the power spectral density (PSD) for each EEG epoch. Then, we obtained the mean PSD per channel and subject from $1 \mathrm{~Hz}$ to $40 \mathrm{~Hz}$. It should be noted that the discrete PSD was computed as the Fourier transform of the biased estimate of the autocorrelation function. Thus, if the raw signal contains $N$ samples, then the autocorrelation function is a vector of length $2 N-1$. Due to this fact, the spectral resolution was $0.1 \mathrm{~Hz}$ in the present study.

$M F$ offers a simple way of summarizing the whole spectral content of the PSD. It is defined as the frequency that comprises $50 \%$ of the power:

$$
0.5{ }_{1 \mathrm{~Hz}}^{40 \mathrm{~Hz}} \operatorname{PSD}(f)==_{1 \mathrm{~Hz}}^{M F} \operatorname{PSD}(f)
$$


It is important to note that the term $M F$ is also known as $50 \%$ spectral edge frequency (SEF50) [23,24]. A parameter strongly related to $M F$ is the mean frequency whose original definition is based on the computation of the spectral centroid. However, previous studies have shown that $M F$ provides a better performance than the mean frequency to characterize spectral changes [25].

\section{B. Individual alpha frequency (IAF)}

$I A F$ quantifies the frequency at which the maximum alpha power is reached. This measure is also called peak frequency. Alpha oscillations are dominant in the EEG of resting normal subjects, with the exception of irregular activity in the delta band and lower frequencies [26]. This issue involves that PSD displays a peak around the alpha band.

The estimation of $I A F$ in the present work was based on the calculation of the $M F$ in the extended alpha band $(4-15 \mathrm{~Hz})$. Thus, problems related to the appearance of several peaks in the considered frequency range or spurious spectral components can be avoided [25]. The equation for calculating IAF parameter is shown bellow:

$$
0.5{ }_{4 \mathrm{~Hz}}^{15 \mathrm{~Hz}} \operatorname{PSD}(f)={ }_{4 \mathrm{~Hz}}^{I A F} \operatorname{PSD}(f)
$$

Both $M F$ and $I A F$ require stationary signals to be appropriately applied. Although EEG recordings are considered non-stationary time series, epochs from background activity up to 10-s duration can be assumed to be wide-sense stationary [27].

\section{Sample Entropy (SampEn)}

SampEn is an embedding entropy that quantifies the signal regularity [28]. It assigns a nonnegative number to a sequence, with larger values corresponding to greater apparent process randomness or serial irregularity, and smaller values corresponding to more instances of recognizable features or patterns in the data. This metric solves some 
problems associated with approximate entropy, a nonlinear method introduced by Pincus to quantify the regularity of time series, initially motivated by applications to relatively short, noisy data sets [29]. SampEn is largely independent of the signal length and displays relative consistency under circumstances where approximate entropy does not. Additionally, SampEn algorithm is simpler than the algorithm used to compute approximate entropy [28].

To calculate SampEn, two input parameters must be specified: a run length $m$ and a tolerance window $r$. It has been suggested to estimate SampEn with parameter values of $m=1$ or 2, and $r$ a fixed value between 0.1 and 0.25 times the SD of the original time series. The algorithm to compute the SampEn is the following [28]:

1) Given a time series, $X=\left(x_{1}, x_{2}, \ldots, x_{N}\right)$, form a set of vectors $X_{m}{ }^{1}, \ldots, X_{m}^{N-m+1}$ defined by $X_{m}{ }^{i}=\left(x_{i}, x_{i+1}, \ldots, x_{i+m-1}\right), i=1, \ldots, N-m+1$.

2) The distance between $X_{m}^{i}$ and $X_{m}{ }^{j}, d\left[X_{m}{ }^{i}, X_{m}{ }^{j}\right]$, is the maximum absolute difference between their respective scalar components:

$$
d\left[X_{m}^{i}, X_{m}^{j}\right]=\max _{k=0, \ldots, m 1}\left|x_{i+k} \quad x_{j+k}\right|
$$

3) For a given $X_{m}^{i}$, count the number of $j(1 \leq j \leq N-m, j \neq i)$, denoted as $B_{i}$, such that $d\left[X_{m}^{i}, X_{m}^{i}\right] \leq r$. Then, for $1 \leq i \leq N-m$,

$$
B_{i}^{m}(r)=\frac{1}{N \quad m 1} B_{i}
$$

4) Define $B^{m}(r)$ as:

$$
B^{m}(r)=\frac{1}{N \quad m}_{i=1}^{N m} B_{i}^{m}(r)
$$


5) Similarly, calculate $A_{i}^{m}(r)$ as $1 /(N-m+1)$ times the number of $j(1 \leq j \leq N-m$, $j \neq i$ ), such that the distance between $X_{m+1}^{j}$ and $X_{m+1}{ }^{i}$ is less than or equal to $r$. Set $A^{m}(r)$ as:

$$
A^{m}(r)=\frac{1}{N \quad m}{ }_{i=1}^{N m} A_{i}^{m}(r)
$$

Thus, $B^{m}(r)$ is the probability that two sequences will match for $m$ points, whereas $A^{m}(r)$ is the probability that two sequences will match for $m+1$ points.

6) Finally, SampEn is estimated by the statistic:

$$
\operatorname{SampEn}(m, r, N)=\ln \frac{A^{m}(r)}{B^{m}(r)}
$$

\section{Lempel-Ziv complexity (LZC)}

LZC algorithm was proposed to evaluate the randomness of finite sequences [30]. It is a nonparametric and simple-to-compute measure of complexity for onedimensional signals that does not require long data segments to be calculated [31]. More complexity in the data produces larger $L Z C$ values.

$L Z C$ analysis is based on a coarse-graining of the measurements. Thus, the EEG time series must be firstly transformed into a finite symbol sequence. In this study, a binary sequence conversion (zeros and ones) was used, since previous studies suggested that this kind of conversion may keep enough signal information [31]. The median value is used as the threshold $T_{d}$, as that partitioning about the median is robust to outliers [32]. By comparison with $T_{d}$, the original time series $X=x(1), x(2), \ldots, x(N)$ is converted into a $0-1$ sequence $P=s(1), s(2), \ldots, s(N)$, with $s(i)$ defined by [31]:

$$
s_{i}=\begin{array}{ll}
0 & \text { if } x_{i}<T_{d} \\
1 & \text { if } x_{i} \quad T_{d}
\end{array}
$$


Afterwards, this string $P$ is scanned from left to right and a complexity counter $c(N)$ is increased by one unit every time a new subsequence of consecutive characters is encountered in the scanning process. Finally, $c(N)$ is normalized in order to obtain a complexity measure independent of the sequence length. For a binary conversion, the upper limit of $c(N)$ is given by $b(N)=N / \log _{2}(N)$, and $c(N)$ can be normalized via $b(N)$ [30]:

$$
C(N)=\frac{c(N)}{b(N)}
$$

The normalized $L Z C, C(N)$, reflects the arising rate of new patterns along with the sequence [28].

\section{E. Statistical analysis}

Initially, a descriptive analysis was carried out to study the results distribution. Normal distribution of the data was evaluated by Kolmogorov-Smirnov and ShapiroWilks tests, whereas Levene test was employed to test for homogeneity of variances. We observed that $M F, I A F$, SampEn and $L Z C$ values did not meet the parametric test assumptions. Therefore, statistical differences between pre-stimulation EEG activity and post-stimulation activity were calculated with the nonparametric Wilcoxon signedrank test (statistical significance $\alpha=0.05$ ). The aforementioned test uses the difference between a paired of related measurements (in our case, before and after therapy) and evaluates whether the distribution of the paired differences deviates from the zero value. Additionally, boxplots were constructed to summarize the changes for each parameter (Post-stimulation - Pre-stimulation) averaged over all channels. Finally, a sensor-level analysis was performed to explore the spatial distribution of each parameter before and after the Snoezelen ${ }^{\circledR}$ intervention, as well as the corresponding $p$-values (Wilcoxon signed-rank test). 


\section{RESULTS}

\section{A. Global analysis}

In a first stage, $M F, I A F$, SampEn and $L Z C$ results were averaged over all EEG channels. Each parameter was computed for each 5 s EEG artifact-free epoch and results were then averaged over all epochs and sensors to obtain a quantitative measure per participant. These results can be seen in Figure 2, where the boxplots corresponding to the differences (Post-stimulation - Pre-stimulation) in (a) $M F$, (b) IAF, (c) SampEn and (d) $L Z C$ values are depicted for TBI, CP and control groups. Our results revealed a slowing of the EEG activity as a consequence of the multi-sensory intervention, as well as a general decrease of irregularity and complexity.

For the TBI group, a significant difference was found with $M F(Z=-2.0251$, $p=0.0428)$. Decreases in IAF, SampEn and $L Z C$ values were also revealed, although these parameters did not show statistically significant differences (IAF: $Z=-1.6117$, $p=0.1070 ;$ SampEn: $Z=-1.6331, p=0.1024 ; L Z C: Z=-1.8509, p=0.0642) . \mathrm{CP}$ group also showed a global decrease for the four methods, but no significant differences were found $(M F: Z=-0.8057, p=0.4204 ; I A F: Z=-0.4355, p=0.6632$; SampEn: $Z=-1.3718, p=0.1701 ; L Z C: Z=-1.5025, p=0.1330)$. Finally, controls showed statistically significant decreases for $\operatorname{LZC}(Z=-2.2428, p=0.0249)$ and some trends towards significance $(p<0.1)$ with the remaining parameters $(M F: Z=-1.7638$, $p=0.0778 ;$ IAF: $Z=-1.9164, p=0.0553 ;$ SampEn: $Z=-1.7202, p=0.0853)$.

\section{PLEASE, DISPLAY FIGURE 2 AROUND HERE}

\section{B. Sensor-level analysis}

In a second step, spatial patterns of $M F, I A F$, SampEn and $L Z C$ values were explored. Thus, these four parameters were computed for each $5 \mathrm{~s}$ EEG artifact-free 
epoch to obtain a quantitative measure per participant and channel. Figure 3 shows the spatial patterns of each parameter before and after the Snoezelen ${ }^{\circledR}$ intervention, as well as the corresponding spatial distribution of significant differences between prestimulation and post-stimulation conditions (Wilcoxon signed-rank test). These results are in agreement with those reported in the global analysis.

Spectral measures revealed a slowing of the EEG activity as a consequence of the therapy (decreased $M F$ and $I A F$ values). This slowing arose both in the extended alpha band and when the complete frequency range was analyzed. For the TBI group, $M F$ decrease was statistically significant in the right central and temporal EEG electrodes. $I A F$ also revealed significant differences in parietal and occipital areas. The spatial distribution of significant differences for $\mathrm{CP}$ group showed a significant $M F$ decrease in both temporal areas and an IAF significant decrease at F8, T6 and O2 EEG locations. Finally, Figure 3 shows a broader pattern of significant decreases for the control group than for both brain-injury groups. Significant differences were found at frontal, parietal and occipital regions with both $M F$ and $I A F$.

Nonlinear measures (SampEn and $L Z C$ ) indicated that post-stimulation condition is characterized by higher regularity and lower complexity values than pre-stimulation. For subjects who have sustained TBI, sensor-level analysis showed a significant reduction of SampEn and $L Z C$ values at electrodes $\mathrm{C} 4$ and O1. For CP group, differences in SampEn values were statistically significant at temporal, parietal and occipital brain regions. Similar findings were reported with $L Z C$. Finally, both nonlinear measures confirm spectral results: significant differences are more widespread in the control group than in brain-injured groups. Statistically significant differences are focused mainly in parieto-occipital area.

PLEASE, DISPLAY FIGURE 3 AROUND HERE 


\section{DISCUSSION}

In this study, we described the changes induced in EEG activity by a Snoezelen ${ }^{\circledR}$ intervention in $18 \mathrm{CP}$ subjects, $18 \mathrm{TBI}$ individuals and 18 controls. For this purpose, EEG activity was analyzed using two conventional spectral measures $(M F$ and $I A F)$ and two nonlinear methods (SampEn and $L Z C$ ). Our results indicate that Snoezelen ${ }^{\circledR}$ intervention elicits a decrease of $M F, I A F$, SampEn and $L Z C$ values. Additionally, statistically significant global decreases of $M F$ in TBI group and of $L Z C$ in control group were found ( $p$-values $<0.05$, Wilcoxon signed-rank test). Our findings support the notion that Snoezelen ${ }^{\circledR}$ therapy affects central nervous system, inducing a slowing of oscillatory activity, as well as a decrease of EEG complexity and irregularity.

Previous studies documented positive effects after the application of multisensory interventions. However, the results of these research works are usually based on subjective and qualitative variables $[7,11,13,14,33,34]$. For instance, the effectiveness of Snoezelen ${ }^{\circledR}$ in dementia care was evaluated watching videorecordings of morning care [34]. In other study, anxiety and depression were assessed using the Hospital Anxiety and Depression scale, whereas quality of life was evaluated using the European Organization for Research and Treatment of Cancer Core 30 questionnaire [33]. In individuals with mental retardation and mental illness, the intervention success was analyzed based on the observation of aggressive acts and self-injurious behaviors [7]. Hauck et al. [14] assessed the usefulness of Snoezelen ${ }^{\circledR}$ in laboring pregnant women on the basis of personal interviews. In sum, Snoezelen ${ }^{\circledR}$ stimulation may be a helpful therapy to deal with disruptive behaviors.

In our study, we have gone a step forward, providing quantitative measures of the changes in EEG activity as a consequence of therapy. The patterns of changes observed using $M F, I A F$, SampEn and $L Z C$ parameters did not necessarily imply that Snoezelen ${ }^{\circledR}$ 
therapy produces neural repair in participants. However, we hypothesize that the changes described in this study are related with higher levels of psychophysiological relaxation of the participants, since similar EEG alterations have been described during different relaxation strategies [35-41]. Nevertheless, other results (e.g. a reduction in delta power and an increase of EEG complexity) contradict our findings [42,43]. Our study revealed that Snoezelen ${ }^{\circledR}$ intervention modifies EEG activity, leading to a slowing of the EEG activity and a reduction of irregularity/complexity values. As we mentioned previously, several relaxation studies agree with these results. For instance, a slowing of the EEG activity has also been observed during relaxation and meditation states [35-38]. These studies reinforce our hypothesis that Snoezelen ${ }^{\circledR}$ stimulation produces deeper states of relaxation in the participants. Previous relaxation researches have reported both a global increase and decrease of EEG power for slow and fast rhythms, respectively [36-38]. These studies confirm that a decline in $M F$ can be associated with relaxation states. In addition, a general increase of EEG activity in theta frequency band has been reported in several researches, independently of the particular meditation technique (for a review, see [35]). Other study proved that relaxation produces significantly greater increases in theta activity in multiple cortical regions compared to listening to music [36]. This neurophysiologic pattern (increase in theta activity) agrees with our IAF results. Nonlinear studies, although less common, have been also applied to characterize relaxation patterns [39-41]. When compared to rest, meditation seems to be accompanied by a complexity decrease, maybe due to the disconnection of irrelevant brain networks for the maintenance of focused internalized attention and inhibition of inappropriate information [39]. In a recent study, a statistically significant reduction in the permutation entropy was found after insight meditation and calming meditation [41]. Natarajan et al. [40] demonstrated that EEG is characterized by a less complex and 
more regular behavior after music and reflexological stimulation, which completely agrees with our SampEn and LZC results. All these studies seem to confirm our hypothesis that Snoezelen ${ }^{\circledR}$ participants are more relaxed after the stimulation.

Our sensor-level results confirmed those reported in the global analysis: the slowing of brain activity and the decrease of complexity and irregularity values. Although statistically significant differences were found at some isolated EEG channels at frontal, central and temporal regions, the main changes between pre-stimulation and post-stimulation conditions were found in occipital and parietal brain areas. These results agree with several studies that tried to identify the brain areas affected by different relaxation-related interventions. In a functional magnetic resonance imaging (fMRI) study [44], changes in the posterior half of the cerebral cortex during natural sensory stimulations were reported. Lazar et al. [45] also employed fMRI to identify the brain areas involved in the relaxation response: temporal lobe, and dorsolateral prefrontal and parietal cortices, among others. Other research work suggested that ZenBuddhist meditation induces changes in the electro-cortical activity of the brain in frontal (alpha-1 frequency band) and occipital (beta band) areas [46]. Lagopoulos et al. [47] revealed that alpha was significantly greater in the posterior area as compared to the frontal region during nondirective meditation. In other study [18], parietal region seems to play an important role for the characterization of the spectral pattern after multi-sensory stimulation. In summary, our sensor-level results revealed particular spatial patterns in brain-injured subjects and controls as a consequence of the Snoezelen ${ }^{\circledR}$ intervention, mainly in posterior regions, which are commonly associated with various aspects of sensory processing [44].

Our spatial analyses also revealed a very important finding: the differences between pre-stimulation and post-stimulation conditions are more widespread in control 
subjects than in both brain-injured groups (statistically significant differences in a higher number of EEG locations). One possible explanation for this fact is related to cognitive deficits in TBI and CP groups, since mild to moderate deficits of attention have been proved in brain-injured subjects in comparison with controls [48]. This lack of attention may diminish the benefits for $\mathrm{CP}$ and TBI groups. The brain lesions suffered by brain-injured subjects can also contribute to reduce the effectiveness of the intervention. On the other hand, the comparison between the two groups of braininjured subjects revealed significant differences at more EEG channels in CP group than in TBI one. Discrepancies between these groups may be due to the severity of the braininjury and/or neural plasticity. Other hypothesis for this finding is that the proposed Snoezelen ${ }^{\circledR}$ intervention is more effective in CP group than in TBI subjects. This interesting result could contribute to design personalized non-pharmacological interventions for each brain-injured cohort. However, this is only an assumption and new researches are needed to confirm the response of each group to multi-sensory intervention.

Finally, some potential confounding factors have to be considered. Firstly, previous studies related to the ingestion of drugs have reported a slowing of EEG activity and a complexity decrease $[49,50]$. In our study, none of the participants were taking any medication that could affect brain activity at the time of EEG recording, so we can discard this issue. Secondly, the detected EEG changes after Snoezelen ${ }^{\circledR}$ intervention appears in other physiological states, such as mental and physical fatigue [51,52]. However, their possible impact is limited, since multi-sensory stimulation sessions lasted only 18 minutes. This fact let us to hypothesize that the alterations are only due to the proposed therapy. Thirdly, only immediate post-session changes in EEG measurements have been analyzed in our study. It is still an open question whether these 
alterations are maintained over time. Therefore, future efforts are needed to explore the longer-term effects. Lastly, a comparison of the effects found in Snoezelen ${ }^{\circledR}$ treatment to other therapeutic environments would be very important in order to define the most effective non-pharmacological strategies.

\section{CONCLUSIONS}

In summary, our research suggests that Snoezelen ${ }^{\circledR}$ intervention elicits significant changes in brain-injured subjects and controls' EEG activity. Specifically, our results revealed a slowing of the oscillatory activity and a decrease of irregularity and complexity. Additionally, the comparison between pre-stimulation and post-stimulation conditions revealed significant differences in TBI, CP and control groups, mainly in occipital and parietal brain areas. This study demonstrates that EEG allows characterizing the changes in brain activity that occur during Snoezelen ${ }^{\circledR}$ intervention. Finally, it is important to note that the methodology proposed in this paper can be useful to describe the effects of this intervention in people with different disorders, as dementia [11], chronic pain [13], autism spectrum disorder [15] and Rett disorder [16]. 


\section{ACKNOWLEDGEMENTS}

This research was supported in part by 'CRE para la Atención a Personas con Grave Discapacidad y Dependencia' (Project 'Evaluación de la terapia multi-sensorial en una sala Snoezelen mediante el análisis de electroencefalogramas en pacientes con daño cerebral'), 'Ministerio de Economía y Competitividad' and FEDER (TEC201453196-R), 'Consejería de Educación de la Junta de Castilla y León’ (VA059U13) and ‘Consejería de Sanidad de la Junta de Castilla y León’ (BIO/VA08/15). The authors would like to thank the brain-injured subjects and the staff at 'CRE para la Atención a Personas con Grave Discapacidad y Dependencia' for participating in this study.

\section{CONFLICT OF INTEREST}

There are no conflicts of interest that could inappropriately influence this research work.

\section{ETHICAL APPROVAL}

This study was approved by the Ethics Committee of the University of León, Spain (reference number \#7-2013). 


\section{AUTHORSHIP RESPONSIBILITY}

- The material in this manuscript is original and contains no matter libelous or otherwise unlawful.

- The manuscript represents valid work and that neither this manuscript nor any other with substantially similar content under my authorship has been published or is being considered for publication elsewhere.

- I have participated sufficiently in the work to take public responsibility for all its content. 


\section{REFERENCES}

[1] Thompson S, Martin S. Making sense of multisensory rooms for people with learning disabilities. Br J Occup Ther 1994;57:341-4.

[2] Fava L, Strauss K. Multi-sensory rooms: Comparing effects of the Snoezelen and the stimulus preference environment on the behavior of adults with profound mental retardation. Res Dev Disabil 2010;31:160-71.

[3] Cuvo AJ, May ME, Post TM. Effects of living room, Snoezelen room, and outdoor activities on stereotypic behavior and engagement by adults with profound mental retardation. Res Dev Disabil 2001;22:183-204.

[4] Matson JL, Bamburg JW, Smalls Y. An analysis of Snoezelen equipment to reinforce persons with severe or profound mental retardation. Res Dev Disabil 2004;25:89-95.

[5] Hogg J, Cavet J, Lambe L, Smeddle M. The use of 'Snoezelen' as multisensory stimulation with people with intellectual disabilities: a review of the research. Res Dev Disabil 2001;22:353-72.

[6] Lancioni GE, Cuvo AJ, O’Reilly MF. Snoezelen: an overview of research with people with developmental disabilities and dementia. Disabil Rehabil 2002;24: $175-84$.

[7] Singh NN, Lancioni GE, Winton AS, Molina EJ, Sage M, Brown S, Groeneweg J. Effects of Snoezelen room, Activities of Daily Living skills training, and Vocational skills training on aggression and self-injury by adults with mental retardation and mental illness. Res Dev Disabil 2004;25:285-93. 
[8] Houghton S, Douglas G, Brigg J, Langsford S, Powell L, West J, Chapman A, Kellner R. An empirical evaluation of an interactive multi-sensory environment for children with disability. J Intellect Dev Disabil 1998;23:267-78.

[9] Withers PS, Ensum I. Successful treatment of severe self injury incorporating the use of DRO, a Snoezelen room and orientation cues. Br J Learn Disabil 1995;23: $164-7$.

[10] Maseda A, Sánchez A, Marante MP, González-Abraldes I, Buján A, MillánCalenti JC. Effects of multisensory stimulation on a sample of institutionalized elderly people with dementia diagnosis: a controlled longitudinal trial. Am J Alzheimers Dis Other Demen 2014;29:463-73.

[11] Moffat N, Barker P, Pinkney L, Garside M, Freeman C. Snoezelen®: An experience for people with dementia. Chesterfield, UK: Rompa; 1993.

[12] Staal JA, Sacks A, Matheis R, Collier L, Calia T, Hanif H, Kofman ES. The effects of Snoezelen (multi-sensory behavior therapy) and psychiatric care on agitation, apathy, and activities of daily living in dementia patients on a short term geriatric psychiatric inpatient unit. Int J Psychiatry Med 2007;37:357-70.

[13] Schofield P. Evaluating Snoezelen for relaxation within chronic pain management. Br J Nurs 2002;11:812-21.

[14] Hauck YL, Summers L, White E, Jones C. A qualitative study of Western Australian women's perceptions of using a Snoezelen room for breastfeeding during their postpartum hospital stay. Int Breastfeed J 2008;3:1-9.

[15] McKee SA, Harris GT, Rice ME, Silk L. Effects of a Snoezelen room on the behavior of three autistic clients. Res Dev Disabil 2007;28:304-16. 
[16] Lotan M, Shapiro M. Management of young children with Rett disorder in the controlled multi-sensory (Snoezelen) environment. Brain Dev 2005;27:S88-94.

[17] Hotz GA, Castelblanco A, Lara IM, Weiss AD, Duncan R, Kuluz JW. Snoezelen: A controlled multi-sensory stimulation therapy for children recovering from severe brain injury. Brain Inj 2006;20:879-88.

[18] Poza J, Gómez C, Gutiérrez MT, Mendoza N, Hornero R. Effects of a multisensory environment on brain-injured patients: assessment of spectral patterns. Med Eng Phys 2013;35:365-75.

[19] McAdams R, Juul SE. Cerebral palsy: prevalence, predictability, and parental counseling. NeoReviews 2011;12:e564-74.

[20] Ghajar J. Traumatic brain injury. Lancet 2000;356:923-9.

[21] Langlois JA, Rutland-Brown W, Wald MM. The epidemiology and impact of traumatic brain injury: a brief overview. J Head Trauma Rehabil 2006;21:375-8.

[22] Parikh S, Koch M, Narayan RK. Traumatic brain injury. Int Anesthesiol Clin 2007;45:119-35.

[23] Rasekhi J, Mollaei MRK, Bandarabadi M, Teixeira CA, Dourado A. Preprocessing effects of 22 linear univariate features on the performance of seizure prediction methods. J Neurosci Methods 2013;217:9-16.

[24] Stewart J, Särkelä M, Koivusalo AM, Wennervirta J, Salmi T, Isoniemi H, et al. Frontal electroencephalogram variables are associated with the outcome and stage of hepatic encephalopathy in acute liver failure. Liver Transpl 2014;20:1256-65. 
[25] Poza J, Hornero R, Abásolo D, Fernández A, García M. Extraction of spectral based measures from MEG background oscillations in Alzheimer's disease. Med Eng Phys 2007;29:1073-83.

[26] Klimesch W. EEG alpha and theta oscillations reflect cognitive and memory performance: a review analysis. Brain Res Rev 1999;29:169-95.

[27] Niedermayer E, Lopes Da Silva F. Electroencephalography: Basic Principles, Clinical Applications and Related Fields. Baltimore, MD: Williams \& Wilkins, 1999.

[28] Richmann JS, Moorman JR. Physiological time-series analysis using approximate entropy and sample entropy. Am J Physiol Heart Circ Physiol 2000;278:H2039_ 49.

[29] Pincus, SM. Approximate entropy as a measure of system complexity. Proc Natl Acad Sci U S A 1991:88;2297-301.

[30] Lempel A, Ziv J. On the complexity of finite sequences. IEEE Trans Inf Theory $1976 ; 22: 75-81$.

[31] Zhang XS, Roy RJ, Jensen EW. EEG complexity as a measure of depth of anesthesia for patients. IEEE Trans Biomed Eng 2001;48:1424-33.

[32] Nagarajan R. Quantifying physiological data with Lempel-Ziv complexity Certain issues. IEEE Trans Biomed Eng 2002;49:1371-3.

[33] Schofield P, Payne S. A pilot study into the use of a multisensory environment (Snoezelen) within a palliative day-care setting. Int J Palliat Nurs 2003;9:124-30. 
[34] van Weert JC, van Dulmen AM, Spreeuwenberg PM, Ribbe MW, Bensing JM. Behavioral and mood effects of snoezelen integrated into 24-hour dementia care. J Am Geriatr Soc 2005;53:24-33.

[35] Cahn BR, Polich J. Meditation states and traits: EEG, ERP, and neuroimaging studies. Psychol Bull 2006;132:180-211.

[36] Jacobs GD, Friedman R. EEG spectral analysis of relaxation techniques. Appl Psychophysiol Biofeedback 2004;29:245-54.

[37] Jacobs GD, Lubar JF. Spectral analysis of the central nervous system effects of the relaxation response elicited by autogenic training. Behav Med 1989;15:125-32.

[38] Knott V, Bakish D, Lusk S, Barkely J. Relaxation-induced EEG alterations in panic disorder patients. J Anxiety Disord 1997;11:365-76.

[39] Aftanas LI, Golocheikine SA. Non-linear dynamic complexity of the human EEG during meditation. Neurosci Lett 2002;330:143-6.

[40] Natarajan K, Acharya UR, Alias F, Tiboleng T, Puthusserypady SK. Nonlinear analysis of EEG signals at different mental states. Biomed Eng Online 2004;3:7.

[41] Vyšata O, Schätz M, Kopal J, Burian J, Procházka A, Jiří K, Hort J, Vališ M. Nonlinear EEG measures in meditation. J Biomed Sci Eng 2014;7:731-8.

[42] Huang HY, Lo PC. EEG dynamics of experienced Zen meditation practitioners probed by complexity index and spectral measure. J Med Eng Technol $2009 ; 33: 314-21$.

[43] Teplan M, Krakovská A, Špajdel M. Spectral EEG features of a short psychophysiological relaxation. Meas Sci Rev 2014;14:237-42. 
[44] Golland Y, Bentin S, Gelbard H, Benjamini Y, Heller R, Nir Y, Hasson U, Malach R. Extrinsic and intrinsic systems in the posterior cortex of the human brain revealed during natural sensory stimulation. Cereb Cortex 2007;17:766-77.

[45] Lazar SW, Bush G, Gollub RL, Fricchione GL, Khalsa G, Benson H. Functional brain mapping of the relaxation response and meditation. Neuroreport 2000;11: $1581-5$.

[46] Huang HY, Lo PC. EEG dynamics of experienced Zen meditation practitioners probed by complexity index and spectral measure. J Med Eng Technol 2009;33: $314-21$.

[47] Lagopoulos J, Xu J, Rasmussen I, Vik A, Malhi GS, Eliassen CF, Arntsen IE, Saether JG, Hollup S, Holen A, Davanger S, Ellingsen $\varnothing$. Increased theta and alpha EEG activity during nondirective meditation. J Altern Complement Med 2009;15:1187-92.

[48] Burg JS, Burright RG, Donovick PJ. Performance data for traumatic brain-injured subjects on the Gordon Diagnostic System (GDS) tests of attention. Brain Inj 1995;9:395-403.

[49] Wackermann J, Lehmann D, Dvorak I, Michel CM. Global dimensional complexity of multi-channel EEG indicates change of human brain functional state after a single dose of a nootropic drug. Electroencephalogr Clin Neurophysiol 1993;86:193-8.

[50] Salinsky MC, Oken BS, Morehead L. Intraindividual analysis of antiepileptic drug effects on EEG background rhythms. Electroencephalogr Clin Neurophysiol 1994; 90:186-93. 
[51] Jap BT, Lal S, Fischer P, Bekiaris E. Using EEG spectral components to assess algorithms for detecting fatigue. Expert Syst App1 2009;36:2352-9.

[52] Liu J, Zhang C, Zheng C. EEG-based estimation of mental fatigue by using KPCA-HMM and complexity parameters. Biomed Signal Process Control 2010;5:124-30. 


\section{TABLES}

Table 1. Clinical data of brain-injured subjects.

\begin{tabular}{|c|c|c|}
\hline Characteristics & TBI & $\mathbf{C P}$ \\
\hline \multicolumn{3}{|l|}{ Severity of the brain-injury (no. of subjects) } \\
\hline Mild/Moderate $(\mathrm{GCS}=9-15 / 15)$ & 14 & 17 \\
\hline Severe $(\mathrm{GCS}=3-8 / 15)$ & 4 & 1 \\
\hline \multicolumn{3}{|l|}{ Time since injury (no. of subjects) } \\
\hline $0.5-4$ years & 5 & 0 \\
\hline $4-8$ years & 8 & 0 \\
\hline$>8$ years & 5 & 18 \\
\hline \multicolumn{3}{|l|}{ Neurosurgery (no. of subjects) } \\
\hline Yes & 9 & 0 \\
\hline No & 9 & 18 \\
\hline \multicolumn{3}{|c|}{ Location of lesion (MRI/CT scan) (no. of subjects) } \\
\hline Left & 7 & 0 \\
\hline Right & 7 & 0 \\
\hline Bilateral & 4 & 18 \\
\hline
\end{tabular}

GCS: Glasgow Coma Scale; MRI: Magnetic Resonance Imaging; CT: Computed Tomography; TBI: Subjects with Traumatic Brain-Injury; CP: Subjects with Cerebral Palsy. 


\section{FIGURE LEGENDS}

Figure 1. Schematic illustration of the session protocol used in the Snoezelen ${ }^{\circledR}$ room.

Figure 2. Boxplots displaying the distribution of the differences (Post-stimulation Pre-stimulation) for (a) $M F$, (b) $I A F$, (c) SampEn, and (d) $L Z C$ values averaged over all EEG channels. $M F$ and $I A F$ results are in $\mathrm{Hz}$, whereas SampEn and LZC are dimensionless parameters. TBI: Traumatic brain-injury group; CP: Cerebral Palsy group; C: Control group.

Figure 3. Topography of each parameter for pre-stimulation and post-stimulation conditions and the corresponding $p$-values for cerebral palsy $(\mathrm{CP})$, traumatic braininjury (TBI) and control (C) groups: (a) $M F$; (b) IAF; (c) SampEn; (d) LZC. MF and $I A F$ results are in $\mathrm{Hz}$, whereas SampEn and $L Z C$ are dimensionless parameters. 